\title{
A novel lens cleaner to prevent water drop adhesions during colonoscopy and esophagogastroduodenoscopy
}

\section{(C) (i) $\odot$}

\author{
Authors

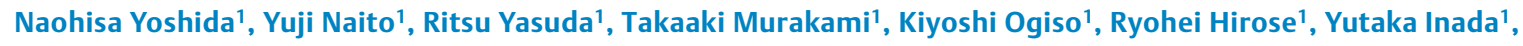 \\ Osamu Dohi ${ }^{1}$, Tetsuya Okayama ${ }^{1}$, Kazuhiro Kamada', Kazuhiko Uchiyama', Takeshi Ishikawa', Osamu Handa', \\ Hideyuki Konishi ${ }^{1}$, Rafiz Abdul Rani ${ }^{2}$, Yoshito Itoh ${ }^{1}$
}

\section{Institutions}

1 Department of Molecular Gastroenterology and Hepatology, Kyoto Prefectural University of Medicine, Graduate School of Medical Science, Kyoto, Japan

2 Gastroenterology Unit, Faculty of Medicine, Universiti Teknologi MARA, Selangor, Malaysia

submitted 20.3.2017

accepted after revision 7.9.2017

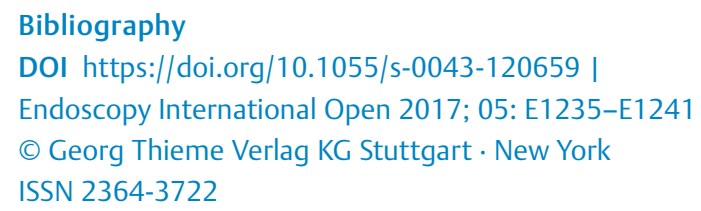

\section{Corresponding author}

Naohisa Yoshida, MD, PhD, Department of Molecular Gastroenterology and Hepatology, Kyoto Prefectural University of Medicine, Graduate School of Medical Science, 465 Kajii-cho, Kawaramachi-Hirokoji, Kamigyo-ku, Kyoto 602-8566, Japan

Fax: +81-75-251-0710

naohisa@koto.kpu-m.ac.jp

\section{ABSTRACT}

Background and study aims Water drop adhesions (WDA) impair endoscopic view during gastrointestinal endoscopy. We developed a novel lens cleaner designed using two types of harmLess surfactants and it is reported to be useful for preventing lens cloudiness during colorectal ESD. In the current study, we examined the ability of it for preventing and removing WDA.
Patients and methods During laboratory experiments, the cleaner (Cleash; Fujifilm Co., Tokyo, Japan and Nagase Medicals Co., Hyogo, Japan) was applied to the endoscopic lens and an air/water device (AWD) (water $200 \mathrm{~mL}$, dimethicone $1 \mathrm{~mL}$, Cleash $1 \mathrm{~mL}$ ). The endoscope was submerged in water 100 times for 5 cycles. Rates of WDA were calculated for various groups (lens and AWD with or without Cleash) and compared to a normal cleaner (SL cleaner). During clinical research, 30 colonoscopies and 30 esophagogastroduodenoscopies were analyzed. For the Cleash group, the cleaner was applied to both lens and AWD. The numbers of WDA and WDA with non-rapid removal were calculated, compared to those of the SL cleaner group.

Results The mean WDA rate for the Cleash setting (lens: Cleash; AWD: Cleash) was $11.0 \%$, which was significantly lower than other settings (lens: SL cleaner; AWD: water, $31.0 \%$; $P<0.001$ ) (lens: Cleash; AWD: water, 19.0\%; $P<$ $0.001)$. Clinical research of colonoscopies indicated that the numbers of WDA (number/15 sec) and WDA with nonrapid removal were 0.38 and 0.17 for the Cleash group and 0.91 and 0.46 for the SL cleaner groups $(P<0.001, P<$ $0.001)$. For esophagogastroduodenoscopies, the results were 0.47 and 0.24 for the Cleash group and 0.54 and 0.42 for the SL cleaner group $(P=0.72, P=0.018)$.

Conclusion A clear and beautiful image without WDA is useful not only for routine endoscopy but also, more importantly, for magnifying endoscopy and other endoscopic treatments. The use of Cleash to lens and AWD showed positive results for preventing and removing WDA during laboratory experiments and clinical research involving CS. Additionally, it also showed positive results for the removal of WDA during EGD.

\section{Introduction}

Water drop adhesions (WDA) and lens cloudiness impair the clarity of the endoscopic view during gastrointestinal endoscopic examinations and treatments, and they are particularly annoying for endoscopists [1]. Difficult visualization is thought to require greater mental concentration for maintaining a safe and accurate procedure. In Japan, we generally use a normal lens cleaner (SL cleaner; Sugiken, Tokyo, Japan) to keep the endoscopic lens clean. Because routine endoscope for finding lesions, magnifying endoscopy, and endoscopic treatments, particularly endoscopic submucosal dissection (ESD) needs 

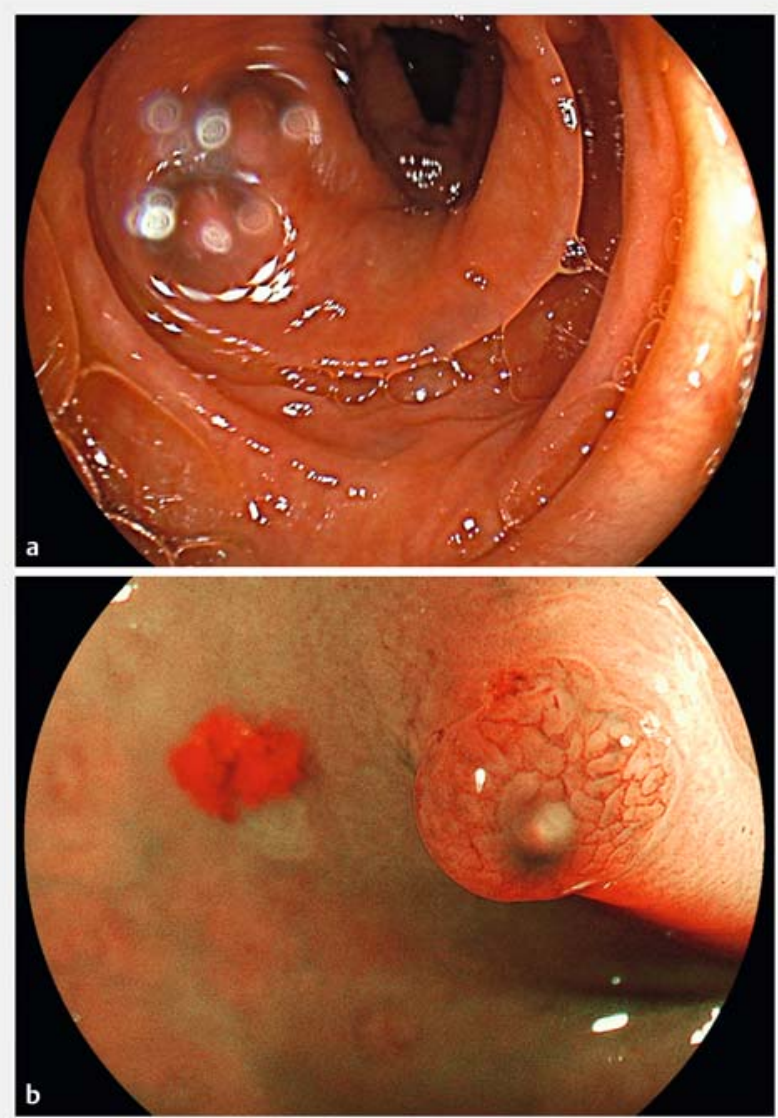

- Fig. 1 Water drop adhesions (WDA) on a colonoscopic lens. a WDA on a routine colonoscope. b WDA on a magnifying colonoscope.

clear endoscopic views [2-6]. However, the efficacy of this lens cleaner is not enough for preventing WDA. Additionally, regarding gastrointestinal endoscopy, there have been no reports of any lens cleaner for preventing WDA.

Recently, we developed a novel lens cleaner (Cleash; Fujifilm Co., Tokyo, Japan and Nagase Medicals Co., Ltd., Hyogo, Japan) that is useful for preventing lens cloudiness during colorectal ESD [7]. The cleaner is designed not only for ESD but also for routine endoscopies, as indicated in the product information. However, to date, there have been no reports about its efficacy for preventing WDA during routine endoscopies. In this study, we examined the ability of this new and unique lens cleaner to prevent WDA during laboratory experiments when used on the endoscopic lens and on air/water devices (AWD). Additionally, we analyzed its ability to prevent and remove WDA with the use of it to both lens and AWD during colonoscopy (CS) and esophagogastroduodenoscopy (EGD) compared to a normal lens cleaner.

\section{Patients and methods}

The novel lens cleaner (Cleash; Fujifilm Co. and Nagase Medicals Co., Ltd.) was prepared using two harmLess, non-ionic surfactants for preventing WDA during CS and EGD ( Fig. 1). We first analyzed its efficacy for preventing WDA during standard use in laboratory experiment 1 ( $\mathbf{F i g} .2$ ). The lens cleaner was applied only to the endoscopic lens ( $\mathbf{F i g}$. 3a). Then, the endoscope was submerged in water 100 times for 5 cycles and the WDA occurrences were calculated for three settings and compared to WDA occurrences with the use of a normal lens cleaner (SL cleaner) as follows: Setting 1, lens without Cleash and AWD with water; Setting 2, lens with Cleash and AWD with water; and Setting 3, lens with SL cleaner and AWD with water. WDA was defined as a water drop on the endoscopic lens that was not removed by about 1 second of air insufflation. Additionally, calculation of WDA was evaluated by endoscopists who did not know whether these cleaners were applied on the lens.

After this experiment, we analyzed a combination of water, dimethicone, and Cleash to determine appropriate AWD ratios. When we applied Cleash to AWD for the first time, we noticed that high concentrations of Cleash cause cumbersome bubbles through the endoscopic channel (2.8-mm endoscopic channel; EG-590WR; Fujifilm Co.) ( Fig. 3b). Therefore, we had to dilute Cleash with water and add dimethicone to prevent bubble formation. Various combinations were evaluated 100 times to assess the optimal ratio in the laboratory experiment 2 ( $>$ Fig. 2 ). Lens cleaner (Cleash) was applied to the tip of the endoscope and to the ADW. The calculation of WDA was evaluated by endoscopists who were blinded to the ratios in each setting. When WDA occurred, the lens was cleaned with 5 seconds of water exposure from the AWD for recoating Cleash. Evaluations were performed about the seven settings described as follows. Setting 4 used $200 \mathrm{~mL}$ water, $0 \mathrm{~mL}$ dimethicone, and $1 \mathrm{~mL}$ Cleash. Setting 5 used $200 \mathrm{~mL}$ water, $1 \mathrm{~mL}$ dimethicone, and $1 \mathrm{~mL}$ Cleash. Setting 6 used $200 \mathrm{~mL}$ water, $5 \mathrm{~mL}$ dimethicone, and $1 \mathrm{~mL}$ Cleash. Setting 7 used $200 \mathrm{~mL}$ water, $1 \mathrm{~mL}$ dimethicone, and $3 \mathrm{~mL}$ Cleash. Setting 8 used $200 \mathrm{~mL}$ water, $5 \mathrm{~mL}$ dimethicone, and $3 \mathrm{~mL}$ Cleash. Setting 9 used $200 \mathrm{~mL}$ water, $1 \mathrm{~mL}$ dimethicone, and $15 \mathrm{~mL}$ Cleash. Setting 10 used $200 \mathrm{~mL}$ water, $5 \mathrm{~mL}$ dimethicone, and $15 \mathrm{~mL}$ Cleash.

After determination of the appropriate ratio for AWD, we analyzed the efficacy of Cleash for preventing WDA by using the accurate amounts on the lens and AWD in the laboratory experiment 3 ( $\mathbf{F i g . 2}$ ). Lens cleaner (Cleash) was applied to the tip of the endoscope and to the ADW. The endoscope was submerged in water 100 times for 5 cycles and WDA were calculated for three different doses of Cleash used for the AWD. Similar to the laboratory experiment 2, Setting 5 was used again (200 mL water, $1 \mathrm{~mL}$ dimethicone, and $1 \mathrm{~mL}$ Cleash). Setting 11 used $200 \mathrm{~mL}$ water, $1 \mathrm{~mL}$ dimethicone, and $3 \mathrm{~mL}$ Cleash. Setting 12 used $200 \mathrm{~mL}$ water, $1 \mathrm{~mL}$ dimethicone, and $0.2 \mathrm{~mL}$ Cleash. Similar to the laboratory experiment 2, When WDA occurred, the lens was cleaned with 5 seconds of water exposure from the AWD. This evaluation was also performed by endoscopists who did not know the ratios. 


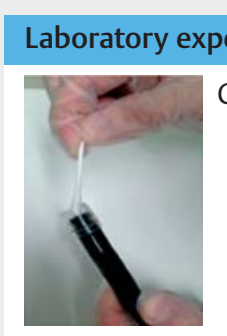

Cleash was applied only to lens for examing the efficacy of Cleash.

Setting 1: nothing

Setting 2: Cleash

Setting 3: SL cleaner

\section{Laboratory experiment 2}

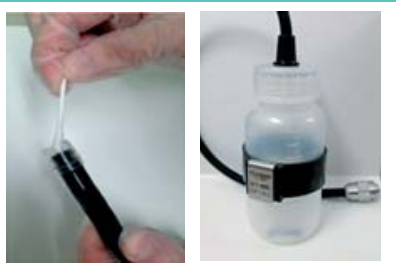

Cleash was applied to lens and AWD for the appropriate setting of AWD.

Setting of AWD

Setting 4: dimethicone $0 \mathrm{ml}+$ Cleash $1 \mathrm{ml}$

Setting 5: dimethicone $1 \mathrm{ml}+$ Cleash $1 \mathrm{ml}$

Setting 6: dimethicone $5 \mathrm{ml}+$ Cleash $1 \mathrm{ml}$

Setting 7: dimethicone $1 \mathrm{ml}+$ Cleash $3 \mathrm{ml}$

Setting 8: dimethicone $5 \mathrm{ml}+$ Cleash $3 \mathrm{ml}$

Setting 9: dimethicone $1 \mathrm{ml}+$ Cleash $15 \mathrm{ml}$

Setting 10: dimethicone $5 \mathrm{ml}+$ Cleash $15 \mathrm{ml}$

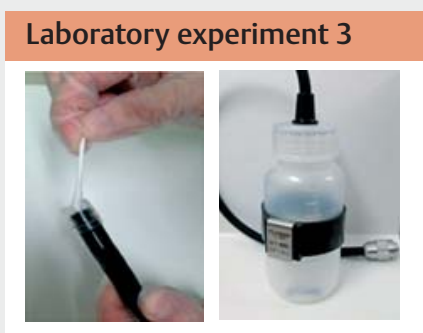

Cleash was applied to lens and AWD for the accurate setting of AWD.

Setting of AWD

Setting 5: dimethicone $1 \mathrm{ml}+$ Cleash $1 \mathrm{ml}$

Setting 11: dimethicone $1 \mathrm{ml}+$ Cleash $3 \mathrm{ml}$

Setting 12: dimethicone $1 \mathrm{ml}+$ Cleash $0.2 \mathrm{ml}$
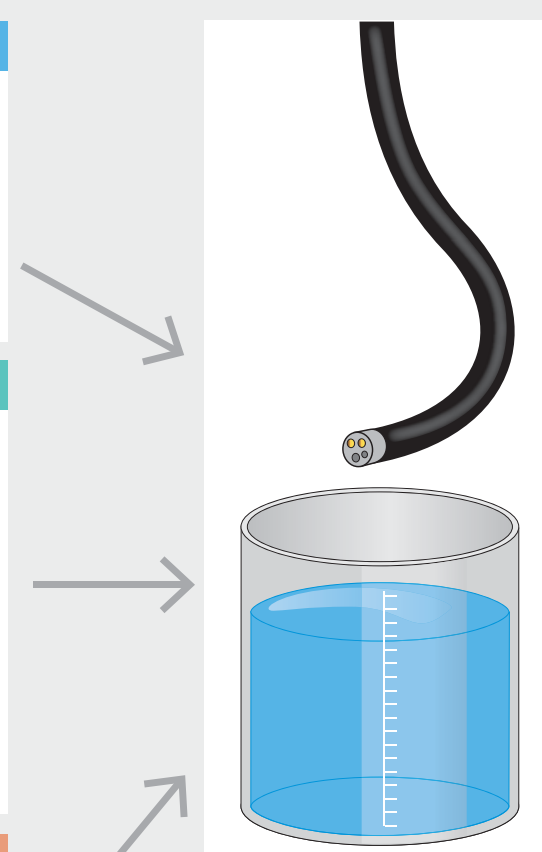

The endoscope was submerged in water 100 times for 5 cycles. Rates of WDA were calculated.

AWD: air/water device WDA: water drop adhesions

- Fig. 2 Three laboratory experiments in our study.
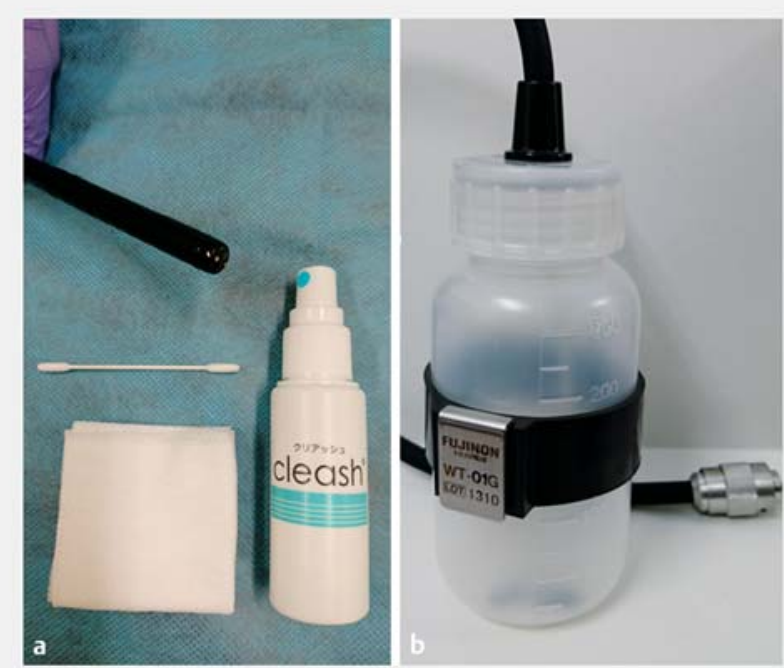

- Fig. 3 Use of Cleash. a Cleash placed on the tip of the endoscope by using a swab or napkin. $\mathbf{b}$ Cleash on the air/water device (AWD) (water $200 \mathrm{~mL}$, dimethicone $1 \mathrm{~mL}$, Cleash $1 \mathrm{~mL}$ ).
We performed clinical research involving CS and EGD at the Kyoto Prefectural University of Medicine in March 2015. For the Cleash group (initial 2 weeks: 15 CS and 15 EGD), Cleash was applied to the endoscopic lens and the AWD (water $200 \mathrm{~mL}$, dimethicone $1 \mathrm{~mL}$, Cleash, $1 \mathrm{~mL}$ ) according to the laboratory experiments ( $\triangleright$ Fig. 3 ). For the SL cleaner group (latter 2 weeks: 15 CS and 15 EGD), the SL cleaner was used only on the endoscopic lens. Because SL cleaner includes some harmful components. All procedures' videos were recorded and WDA and WDA with non-rapid removal were calculated by endoscopists. These numbers were divided by the endoscopic procedure time and evaluated (number/15 sec) for the Cleash group and SL cleaner group regarding CS and EGD. The endoscopists did not perform these endoscopies and were blinded to when Cleash was used. Additionally, endoscopists who performed CS and EGD evaluated the status of WDA and WDA with non-rapid removal subjectively for the Cleash group and SL cleaner group, using a grading system with scores from 1 to 4 (1, poor; 2, fair; 3, good; 4, excellent). During clinical research, we used a single-channel EGD endoscope (EG-590WR and EG-L590WR; Fujifilm Co.); and, for CS, we used a single-channel medium-length endoscope (EC-L590ZP and EC-L590ZW; Fujifilm Co.) with a minimal length of transparent hood. 
All patients provided written informed consent to undergo CS and EGD. This study received approval from the ethics committees of Kyoto Prefectural University of Medicine and was performed in accordance with the Declaration of Helsinki. In addition, it is registered on the University Hospital Medical Information Network Clinical Trials Registry (UMIN-CTR; number UMIN000015584).

\section{Components of the novel cleaner}

As previously reported, the novel lens cleaner (Cleash) was designed using two types of non-ionic, harmless surfactants (polyglycerol fatty acid esters with high hydrophile-lipophile balance [HLB] or low HLB) [7]. This cleaner has been available for purchase in Japan since 2015. Before this study using AWD, we confirmed that the cleaner did not affect the function of the endoscope, AWD, or various endoscopic accessories (snare, knife, injection needle, etc.) with the help of Fujifilm Co. The toxicity of the cleaner was evaluated by a basic experiment involving rats administered $50-\mathrm{mL}$ Cleash; significant changes in the toxicity index were not observed [7]. Cleash also did not affect gastric ulcer in rat models. Our new method using AWD involved the possibility of a small amount of Cleash entering the patient's gastrointestinal tract. The maximum amount of Cleash was estimated to be 1 to $2 \mathrm{~mL}$ during one CS and EGD procedure. The amount of polyglycerol esters of fatty acids in $2 \mathrm{~mL}$ Cleash was $0.4 \mathrm{~g}$. The limitation of polyglycerol esters of fatty acids for human toxicity is estimated within $20 \mathrm{~g}$ according to Japanese Food Sanitation and basic research. Thus, we could use it for AWD with the technical advice of Nagase Medicals Co. However, SL cleaner is made of harmful components such as n-alkylbenzenesulfonate and poly(oxyethylene) alkyl ether; therefore, we did not use it for AWD.

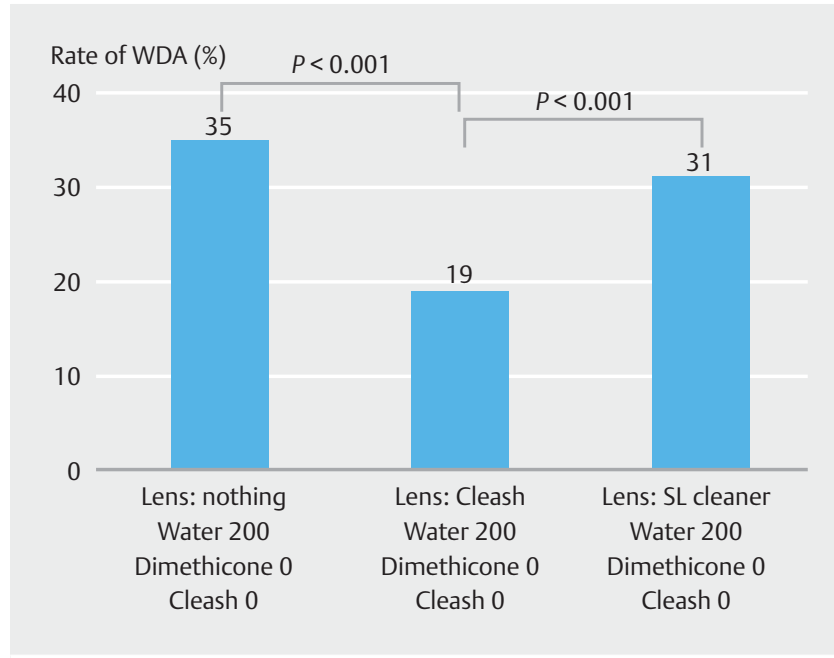

Fig. 4 Mean rate of water drop adhesions (WDA) with use of Cleash on an endoscopic lens compared to no cleaner and SL cleaner.

\section{Statistical analysis}

The sample size for clinical research (15 EGD cases and 15 CS cases in the Cleash group) was determined according to laboratory experiments. We predicted that Cleash could decrease WDA by $25 \%$ compared to non-use of Cleash. Using a Wilcoxon signed rank test, the $\alpha$ error was 0.05 and the $\beta$ error was 0.2 . Therefore, the minimum sample size was calculated as 13 . Statistical analyses were performed using the Mann-Whitney $U$ test and the chi-square test (SPSS version 22.0 for Windows; IBM Japan, Ltd., Tokyo, Japan). Categorized variables such as WDA grade according to endoscopists were analyzed using the

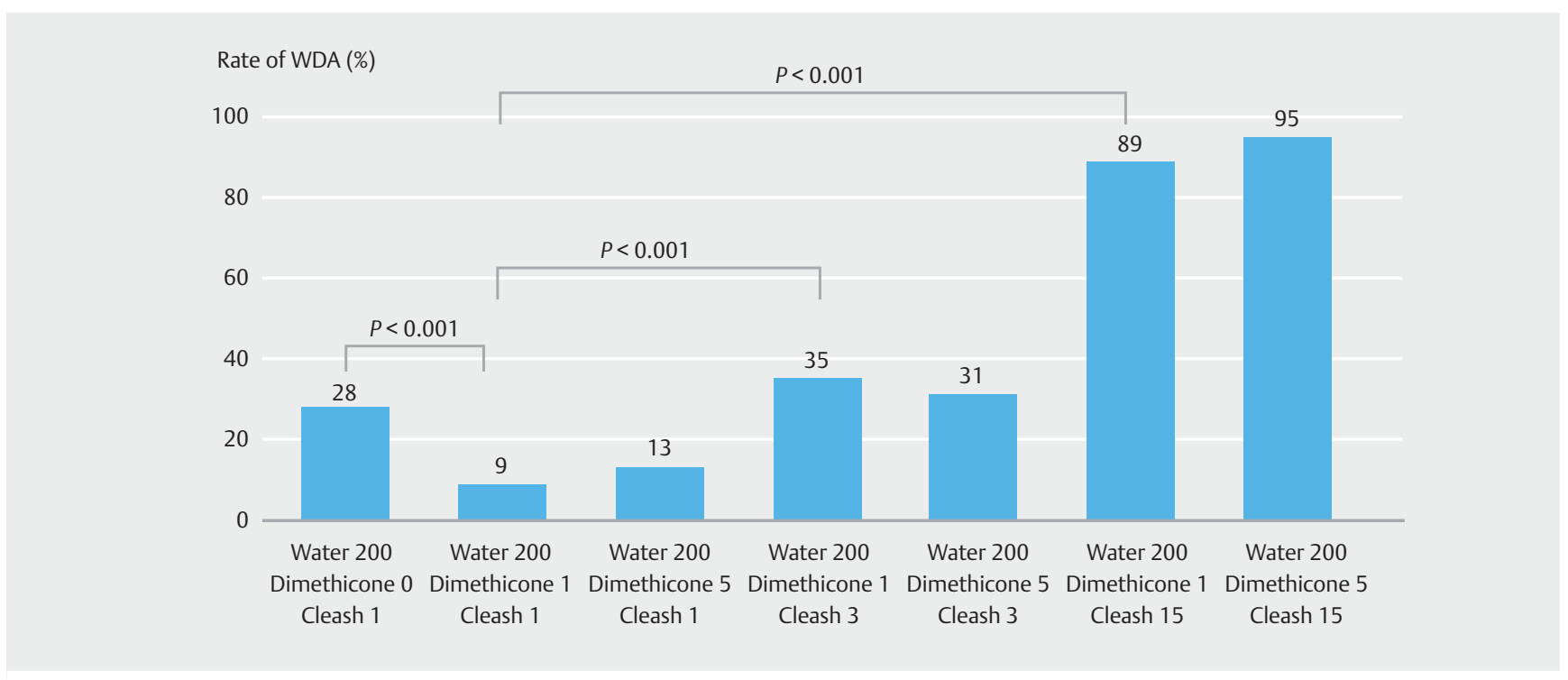

Fig. 5 Mean rate of water drop adhesions (WDA) with use of Cleash for both the endoscopic lens and air/water device (AWD). 


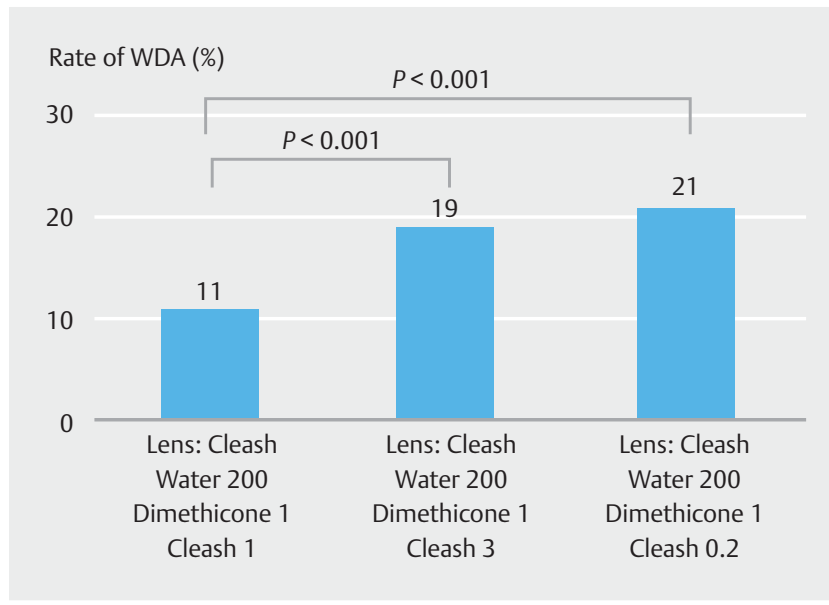

- Fig. 6 Mean rate of water drop adhesions (WDA) with accurate amounts of use of Cleash for both the endoscopic lens and air/ water device (AWD) (water $200 \mathrm{~mL}$, dimethicone $1 \mathrm{~mL}$, Cleash $0.2-3 \mathrm{~mL})$.

Mann-Whitney $U$ test. A $P$ value $<0.05$ was considered statistically significant.

\section{Results}

The laboratory experiment 1 to determine the efficacy of Cleash on the endoscopic lens showed that the mean WDA rates were $35 \%$ (lens: nothing, AWD: water), 19\% (lens: Cleash, AWD: water), and $31 \%$ (lens: SL cleaner, AWD: water) $(P<$ 0.001) ( Fig.4). Occurrences of WDA on the use of Cleash to AWD were evaluated for the seven settings in the laboratory experiment 2 . The rate of WDA using water $200 \mathrm{~mL}$, dimethicone
$1 \mathrm{~mL}$, and Cleash $1 \mathrm{~mL}$ was $9 \%$, which was significantly lower than the rates for the other three ratios: $28 \%$ (water $200 \mathrm{~mL}$, dimethicone $0 \mathrm{~mL}$, Cleash $1 \mathrm{~mL} ; P<0.001$ ), 35\% (water $200 \mathrm{~mL}$, dimethicone $1 \mathrm{~mL}$, Cleash $3 \mathrm{~mL} ; P<0.001$ ), and $89 \%$ (water $200 \mathrm{~mL}$, dimethicone $1 \mathrm{~mL}$, Cleash $15 \mathrm{~mL}$; $P<0.001$ ) ( $\mathbf{F i g . 5}$ ). Additionally, the use of Cleash to the endoscopic lens with the three different doses of Cleash for the AWD were examined in the laboratory experiment 3. The WDA rate for water $200 \mathrm{~mL}$, dimethicone $1 \mathrm{~mL}$, and Cleash $1 \mathrm{~mL}$ was $11 \%$, which was significantly better than those of the other two ratios (19\% for water $200 \mathrm{~mL}$, dimethicone $1 \mathrm{~mL}$, Cleash $3 \mathrm{~mL} ; 21 \%$ for water $200 \mathrm{~mL}$, dimethicone $1 \mathrm{~mL}$, Cleash $0.2 \mathrm{~mL} ; P<0.001$ ) (> Fig.6). In addition, that rate was better than the mean WDA rates for Cleash (19.0\%; $P<0.001)$ or SL cleaner $(31.0 \%$; $P<0.001)$ and only water on the AWD ( $\triangleright$ Fig. 4 ).

Regarding the clinical research of CS, the numbers of WDA (number/15 sec) and WDA with non-rapid removal (number/ $15 \mathrm{sec}$ ) were 0.38 and 0.17 , respectively, in the Cleash group and 0.91 and 0.46 , respectively, in the SL cleaner group $(P<$ 0.001 and $P<0.001$ ) ( $\vee$ Fig. 7). For the research of EGD, the results were 0.47 and 0.24 , respectively, in the Cleash group and 0.54 and 0.42 , respectively, in the SL cleaner group $(P=0.72$ and $P=0.018)$.

Subjective evaluations by endoscopists of CS and EGD are shown in $>$ Table 1. The WDA grade for CS was significantly better in the Cleash group than in the SL Cleaner group $(3.4 \pm 0.3$ vs. 2.6 $\pm 0.7 ; P<0.001)$. Additionally, the grade of WDA with non-rapid removal was also significantly better in the Cleash group than in the SL cleaner group (3.6 0.5 vs. $2.6 \pm 0.8 ; P<$ $0.001)$. On the other hand, for EGD, only the WDA grade was significantly better in the Cleash group than SL cleaner group (3.3 \pm 0.7 vs. $2.8 \pm 0.9 ; P=0.047)$.

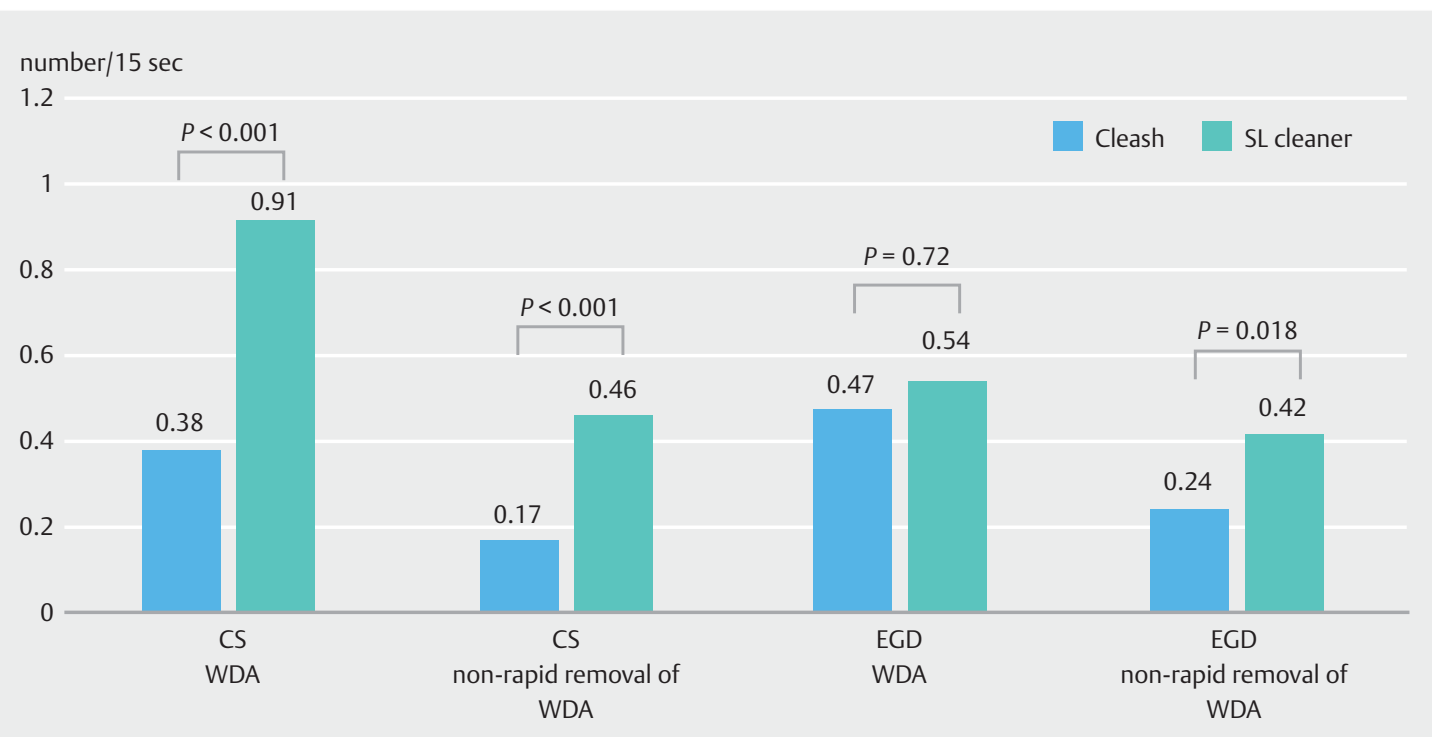

Fig. 7 Numbers of water drop adhesions (WDA) (number/15 sec) and WDA with non-rapid removal (number/15 sec) using a lens and AWD. Comparison was performed between Cleash (lens and AWD) and SL cleaner (only lens) during clinical research involving 30 colonoscopies (CS) and 30 esophagogastroduodenoscopies (EGD). 
- Table 1 Subjective evaluations of WDA and WDA with non-rapid removal during CS and EGD.

\begin{tabular}{|c|c|c|c|c|c|c|c|}
\hline CS & $\begin{array}{l}\text { Cleash } \\
n=15\end{array}$ & $\begin{array}{l}\text { SL cleaner } \\
n=15\end{array}$ & $P$ value & EGD & $\begin{array}{l}\text { Cleash } \\
n=15\end{array}$ & $\begin{array}{l}\text { SL cleaner } \\
n=15\end{array}$ & $P$ value \\
\hline WDA grading & $3.4 \pm 0.3$ & $2.6 \pm 0.7$ & $<0.001$ & WDA grade & $3.3 \pm 0.7$ & $2.8 \pm 0.9$ & 0.047 \\
\hline $\begin{array}{l}\text { WDA with non-rapid removal } \\
\text { grade }\end{array}$ & $3.6 \pm 0.5$ & $2.6 \pm 0.8$ & $<0.001$ & $\begin{array}{l}\text { WDA with non-rapid removal } \\
\text { grade }\end{array}$ & $3.0 \pm 1.0$ & $3.0 \pm 1.2$ & 0.89 \\
\hline
\end{tabular}

\section{Discussion}

In the current study, prevention and removal of WDA on the endoscopic lens during CS significantly improved with this novel cleaner. In addition, removal of WDA during EGD improved. Our unique, original method of applying the novel cleaner to both the lens and the AWD showed a positive effect on prevention and removal of WDA.

Previous studies showed the effect of oolong tea for preventing cloudiness of the endoscopic lens during transnasal EGD [8]. Saponin is a surfactant in oolong tea that is thought to be useful for keeping the endoscopic lens clean [9]. However, in our experience, the effect of oolong tea was limited. To our knowledge, there are no other reports regarding other gastrointestinal endoscopic lens cleaners. Therefore, this is the first study about an original endoscopic lens cleaner applied to the endoscopic lens and the AWD. Additionally, strong surfactants are effective for preventing lens cloudiness but are harmful to the human body. Therefore, we used only safe and effective types of surfactants to create Cleash.

We previously reported the efficacy of Cleash for colorectal ESD [7]. The rate of lens staining due to coagulated debris and mucus significantly decreased in the Cleash group compared to SL cleaner group $(14.1 \%$ vs. $33.0 \% ; P<0.01)$. In that study, Cleash was not used on the AWD; it was applied only to the endoscopic lens and transparent hood. In addition to lens staining prevention, we reported other unique and novel uses of Cleash for severe lens staining [7]. By pressing the endoscopic hood against the mucosa, an enclosed space was created and the cleaning solution was injected via the endoscopic channel and maintained for 30 seconds. Using this method, the lens became clear in all seven cases of severe lens staining, thereby negating the need to remove the endoscope for lens cleaning and allowing uninterrupted ESD for all cases. In the current study, we proved the efficacy of Cleash both on the endoscopic lens and on the AWD. Therefore, we think that lens staining and cloudiness during ESD are prevented more by this unique method.

In laparoscopy, condensation on the scope lens occurs due to the difference between room temperature and intra-abdominal temperature. An anti-fog solution (LiNa Clear Sight; Lina Medical Aps, Glostrup, Denmark) is available. Heating the lens using methods such as a warming bath and thermos flask are also helps prevent condensation of the lens $[10,11]$. We performed a trial of these methods for the gastrointestinal endo- scope, but they were not proven useful for preventing WDA. Therefore, we believe Cleash may be the solution to the problem of condensation on the laparoscopic lens because of the mounting evidence presented here. However, it does involve some problems such as health insurance licensing and the need for strict sterilization before use.

There were some limitations in our study. It was a singlecenter study and performed by reviewing videos to calculate WDA in clinical research. We only used Fujifilm endoscopic system in this study.

\section{Conclusion}

In conclusion, a clear and beautiful image without WDA is useful not only for routine endoscopy but also, more importantly, for magnifying endoscopy and other endoscopic treatments. Use of Cleash to lens and AWD showed positive results for preventing and removing WDA during laboratory experiments and clinical research involving CS. In addition, it also showed positive results for removal of WDA during EGD.

\section{Acknowledgements}

We thank all other members of the Department of Molecular Gastroenterology and Hepatology at Kyoto Prefectural University of Medicine for their help with this study.

\section{Competing interests}

Cleash in this study was provided by Nagase Medicals Co., Ltd. Yoshito Itoh is affiliated with Fujifilm Medical Co., Ltd. The other authors have no conflicts of interest to declare. Other than those declared previously, no financial support or relationships exist that may pose a conflict of interest for our manuscript.

\section{References}

[1] Kobayashi E, Kakuda M, Tanaka Y et al. A novel device for cleaning the camera port during laparoscopic surgery. Surg Endosc 2016; 30: 330-334

[2] Yoshida N, Hisabe T, Inada Y et al. The ability of a novel blue laser imaging system for the diagnosis of invasion depth of colorectal neoplasms. J Gastroenterol 2014; 49: $73-80$ 
[3] Kudo S, Hirota S, Nakajima T et al. Colorectal tumours and pit pattern. J Clin Pathol 1994; 47: 880 - 885

[4] Yoshida N, Hisabe T, Hirose R et al. Improvement in the visibility of colorectal polyps by using blue laser imaging. Gastrointest Endosc 2015; 82: 542-549

[5] Saito Y, Uraoka T, Yamaguchi Y et al. A prospective, multicenter study of 1111 colorectal endoscopic submucosal dissections (with video). Gastrointest Endosc 2010; 72: 1217-1225

[6] Sano Y, Tanaka S, Kudo SE et al. Narrow-band imaging (NBI) magnifying endoscopic classification of colorectal tumors proposed by the Japan NBI Expert Team. Dig Endosc 2016; 28: 526 - 533

[7] Yoshida N, Naito Y, Hirose R et al. Risk of lens cloudiness during colorectal endoscopic submucosal dissection \& ability of a novel lens cleaner to maintain and restore endoscopic view. Dig Endosc 2015; 27: $609-617$

[8] Komazawa Y, Amano Y, Yuki M et al. Oolong tea is useful for lens cleansing in transnasal small-caliber esophagogastroduodenoscopy. Endoscopy 2010; 42: 104 - 108

[9] Song S, Zhu L, Zhou W. Simultaneous removal of phenanthrene and cadmium from contaminated soils by saponin, a planet-derived biosurfactant. Environ Pollut 2008; 156: $1368-1370$

[10] Van Deurzen DF, Mannaerts GH, Jakimowicz JJ et al. Prevention of lens condensation in laparoscopic surgery by lens heating with a thermos flask. Surg Endosc 2005; 19: $299-300$

[11] Brown JA, Inocencio MD, Sundaram CP. Use of warming bath to prevent lens fogging during laparoscopy. J Endourol 2008; 22: 213-214

\section{CORRECTION}

Yoshida N, Naito Y, Yasuda R et al. A novel lens cleaner to prevent water drop adhesions during colonoscopy and esophagogastroduodenoscopy.

Endoscopy International Open 2017; 05: E1235-E1241. DOI: 10.1055/s-0043-120659

In the above mentioned article was a value of the second bar in figure 5 incorrect. Correct is: Dimethicone 1. 